\title{
Randomized controlled trial on the effect of
} Internet-based Cognitive Behavioral Therapy with Real-Time Therapist Support Via a Video Conference for patients with chronic pain: A study protocol

Kayoko Taguchi ( $\square$ kurogane15@icloud.com )

https://orcid.org/0000-0003-3077-7200

Noriko Numata

Chiba University

Kana Kutsuzawa

Chiba University

Kensuke Yoshimura

Chiba University

Eiji Shimizu

Chiba University

\section{Study Protocol}

Keywords: individual cognitive behavioral therapy, chronic pain, randomized controlled trial, telemedicine

Posted Date: January 23rd, 2020

DOI: https://doi.org/10.21203/rs.2.21702/v1

License: (c) (i) This work is licensed under a Creative Commons Attribution 4.0 International License.

Read Full License 


\section{Abstract}

Background: Chronic pain, which persists for at least 12 weeks, is a serious medical condition that not only causes numerous physical and mental conditions, but also affects a person's entire life. Previous studies concerning cognitive behavioral therapy for patients with chronic pain have shown low to intermediate effectiveness. This paper describes the study protocol for a randomized controlled trial (RCT) to evaluate the effectiveness of our newly developed Internet-based Cognitive Behavioral Therapy with Real-Time Therapist Support Via a Video Conference (VCBT) as an adjunct to usual care compared with only usual care for chronic pain. Methods: The RCT comprises two parallel groups (VCBT + usual care and usual care alone) of 20 participants each $(n=40)$ who were diagnosed with chronic pain. We aim to evaluate the effectiveness of the intervention over 16 weeks. The primary outcome is pain intensity, which will be assessed by the Numerical Rating Scale (NRS) at week 16 . Secondary outcomes are pain catastrophic perception and daily life impairment degree, each assessed by the Pain Catastrophizing Scale (PCS) and Pain Disability Assessment Scale (PADS). In addition, anxiety, depression, and quality of life will be assessed. All measures will be assessed at weeks 1 (baseline), 8 (middle), and 16 (post). Discussion: This study aims to demonstrate the effectiveness of Internet-based CBT with Real-Time Therapist Support Via a Video Conference for patients with chronic pain. The findings of this study will show a feasibility and safety of online CBT for chronic pain, valuable addition to the treatment options for these patients. And we expect that many therapists will be able to universally provide CBT of comparable quality in the treatment of chronic pain by using the CBT protocol which we have developed.

\section{Background}

Pain plays an important role for human beings. It functions to inform us of injury or disease, encourages us to seek medical care, and contributes to the healing process by promoting rest and recovery. Pain also reminds us of harmful past events and situations, teaches us what to avoid in the future, and motivates us to terminate the cause of the pain. This characterizes so-called acute pain, and its function usually ends when an injury heals, or a disease is cured. On the other hand, chronic pain, which persists for more than 12 weeks, is physiologically unnecessary. Chronic pain of unknown origin increases stress levels, which also trigger other diseases, such as insomnia and depression. According to the latest version of the World Health Organization (WHO) International Disease Classification version 11 (ICD-11) ${ }^{1}$ published in 2018, chronic pain is defined as an unpleasant sensory and emotional experience that persists or recurs for longer than 3 months, is associated with actual or potential tissue damage, or is described in terms of such damage. Chronic pain is categorized into the following 7 groups: chronic primary pain, chronic cancer pain, chronic postsurgical and posttraumatic pain, chronic neuropathic pain, chronic headache and orofacial pain, chronic visceral pain, and chronic musculoskeletal pain. Optional specifiers for each diagnosis record evidence of psychosocial factors and the severity of the pain. Pain severity can be graded based on pain intensity, pain-related distress, and functional impairment ${ }^{2}$.

Limited worldwide reports have proven that chronic pain affects an estimated $20-50 \%$ of people $2,3,4,5$ and accounts for $15-20 \%$ of physician visits. Medical treatment, physiotherapy, psychotherapy and other 
medical treatments for chronic pain should be comprehensively administered. However, under the current circumstances, patients often only receive treatment from the department they visit. Therefore, it is difficult to determine if patients with chronic pain receive effective treatment.

\section{Standard treatment for chronic pain}

Many patients with chronic pain receive several treatments ${ }^{6}$. Standard treatment for chronic pain begins with low-level invasive treatment centered on oral drugs, and builds gradually to highly invasive treatment. Specifically, the first medical treatment may include psychosomatic treatment and rehabilitation. Furthermore, various surgical and orthopedic treatments are considered. In pharmacotherapy, tricyclic antidepressants (TCA) and serotonin noradrenaline reuptake inhibitors (SNRI) are used as first-line drugs, or gabapentin (Pregabalin) and peripheral neuropathic pain remedy. When these prove ineffective, an antiepileptic drug is considered, but the evidence for success is minimal. Next, various nerve block injections, laser irradiation in anesthetic, psychotherapy (i.e., relaxation), counseling, or psychiatric psychoeducation will be attempted. Chronic pain should not be treated with one method alone. The basic treatments are multidisciplinary, and include drug therapy, physical therapy, psychotherapy, etc. However, as multidisciplinary therapy is expensive ${ }^{6}$, the development of cost-effective therapeutic methods is urgent.

\section{CBT for chronic pain}

Cognitive Behavioral therapy (CBT) is a structural psychotherapy that encourages transformation of a patient's cognition and behavior, and it can directly intervene in "excessive ideas, concerns, emotions, and behaviors related to physical symptoms," which are major factors of chronic pain. CBT is already recognized as an effective and recommended treatment for chronic pain. According to the systematic review revealing the effectiveness of $\mathrm{CBT}$ for chronic pain based on 25 trials reported that there is a low level of evidence (effect size $0.28-0.4$ ) compared to the active treatment group. In addition, there is moderate evidence (effect size mean 0.5 ) in all items compared to the waiting group ${ }^{7}$. In a recent report, an integrative systematic review revealed that $\triangle$ CBT reduced pain intensity in $43 \%$ of 35 trials. Efficacy of online and in-person formats were comparable, and military veterans and individuals with cancer-related chronic pain were not sufficiently studied ${ }^{8}$. For example, clinical research on CBT for lower back pain, which is the most commonly presenting form of chronic pain, showed that clinical improvement of pain intensity and disability due to pain was comparable to the usual treatment ${ }^{9}$. The problem seems to lie in the fact that effect size of $\mathrm{CBT}$ for chronic pain is limited to low to moderate evidence in many studies ${ }^{10}$. Furthermore, there are insufficient studies showing that $C B T$ is more effective as a treatment for chronic pain.

\section{Internet based CBT for chronic pain}

Remote treatment via the internet has been increasing and has demonstrated its effectiveness ${ }^{11,12,13,14}$. Since internet based CBT is easy to access and can be administered at home, it can be considered a convenient intervention for patients who have difficulty traveling to hospitals due to pain. Concerning 
CBT for chronic pain, similar clinical research reports about using internet $C B T$ are increasing ${ }^{15,16,17}$. A systematic review of Internet-based CBT for chronic lower back pain suggested reducing catastrophizing cognition and improving patient attitudes ${ }^{18}$. However, as considered in this review, there is limited clinical research on this topic necessitating further research to be performed.

\section{Methods/design}

\section{Aim of study}

Our present study was undertaken to verify the effectiveness of the Internet-based Cognitive Behavioral Therapy with Real-Time Therapist Support Via a Video Conference (VCBT) which we have carefully challenged. This study was designed as a prospective randomized open-labelled pilot trial with two parallel 16-week intervention groups: a vCBT group that also received treatment as usual and a continuing TAU group alone (Fig. 1).

Our hypothesis considers that the vCBT group will show more effectiveness than the continued usual care group in both pain intensity and other pain-related outcomes. In previous studies, CBT has shown improvement over usual care alone in disability of daily life and catastrophizing thought due to pain. However, the degree of effectiveness is insufficient, and we want to demonstrate greater effectiveness using this program.

\section{Participants and recruitment}

As all participants will continue to be treated by their general practitioners, participants will be required to obtain permission from them prior to study enrolment. Inclusion and exclusion criteria show Participant recruitment was conducted through posters and leaflets placed at medical institutions in Chiba Prefecture and through web-based and newspaper advertisements from April 2018 to November 2019. Further, this study will be announced to outpatients in the Department of Orthopedics and Pain Anesthesiology in Chiba University Hospital.

\section{Eligibility procedure for participation and diagnosis}

Participants are requested to keep a record of pain assessment for a week and to submit this record at the initial visit. To determine eligibility, participants will visit a doctor and receive an explanation about the risks, benefits, and expected commitment to the study. Finally, they will be requested to provide informed consent. After obtaining informed consent, a screening test based on inclusion and exclusion criteria will be conducted by a clinical psychologist or nurse (Table 1).

\section{Randomization and case registration}

After completing the baseline assessment, eligible participants will be randomly assigned to either the TAU group or the VCBT group, using the minimization method, ensuring a balance in pain scores and gender. As the allocation adjustment factor, the pain score on NRS is allocated at more than 6.3 or less ${ }^{19}$. 


\section{Description of the intervention}

Both usual care and vCBT groups will undergo 16 weeks of intervention. In both groups, patients answer questions consisting of primary and secondary outcomes at 1 (baseline), 8 (middle), and 16 (post) weeks after the intervention, and mail to the researcher.

\section{Internet-based CBT for chronic pain program}

Internet-based CBT programs for chronic pain generally consist of 8-12 intervention sessions. Contents are usually psychological education for pain, case formulation for searching the vicious circle of pain, cognitive reconstruction and relaxation as a self-control skill over pain, etc. We have added four experimental sessions, including one based on the latest knowledge about brain science and psychology, to this program to further improve its impact ${ }^{8}$ (Table 2). We have incorporated four new sessions that have not been used in CBT protocols for chronic pain so far.

Tactile attention-shift training(session4); The patient notices the "attention bias," given to their painful body parts and needs to correct the attention imbalance. There is a hypothesis that pain can be relieved through promoting secretion of oxytocin through the therapist's gentle touching of the affected body part. This is called Science Touch and suggests relief of chronic pain ${ }^{26}$. Attention shift training occurs while paying attention to the therapeutic effect of "touching." It takes practice to make it possible to freely shift attention from the painful body part to the part without pain.

Memory work using the peak end rule(session10); Chronic pain patients tend to regard a pain experience as a much stronger pain experience than in reality, because pain memory acts as a traumatic memory. Kahneman et al. showed in empirical studies that unpleasant experiences like pain are memorized as the average of the strength of the pain peak and the strength of the end. He suggests that the average of an event's most unpleasant impression and best impression will remain as memories ${ }^{25}$. However, for chronic pain patients, intense pain experiences create strong impressions such that the good impression made when the pain is relieved is considered unclear. Therefore, the average of impressions concerning pain is high and is preserved as an intense pain experience. It is expected that pain will be relieved by recollecting the memories of intense pain in detail and rewriting them to objective memory.

Sharpening behavioral image training (session11); This has been applied to body image training used in sports science or rehabilitation medicine after stroke ${ }^{20,21}$. Patients practice until they can clearly imagine moving their painful body part. Fixing the visual image into memory may increase brain plasticity and lead to recovery of chronic pain.

Video feedback including mirror therapy(session12);In mirror therapy used as treatment for phantom limb pain $^{22}$, the patient observes movement of the healthy side as reflected in the mirror. Through this visual recognition, the brain understands as if it is the amputated side that is moving, and the pain will be alleviated $^{23,24}$ If the chronic pain is regarded as a state in which sense and motor contact to the brain is 
severely disconnected as with phantom limb pain, it is expected that the pain will be alleviated by continuing to observe the video image where the patient's own painful body part is moving.

The CBT intervention will consist of 16 weekly 50 -minute sessions using the internet video conference system. We developed the manual based on the existing published resources ${ }^{27}$, as well as on materials we used in a prior study for anxiety disorder. In this study, we adopt Cisco WebEx as the internet conference system. This system has acquired ISO 27001 certification. Countermeasures against unauthorized access, information leakage, etc. have been taken, and safety problems are also being cleared. If the therapist or the participant finds it impossible to continue due to adverse events, etc., interruptions of up to 30 days can be made only once in all sessions.

\section{Usual care}

The usual care group requires regular visits at least once every eight weeks, with or without drug therapy at the start of study participation.

\section{Therapist}

The therapist should be medically qualified, such as a doctor, nurse, clinical psychologist, mental health care worker, and have finished the basic CBT training by the CHIBA-IAPT, workshop of the Anxiety Society, or the equivalent. In addition, the therapist should have experience implementing CBT under supervision. The therapist will video record every session during the intervention period and will manage the quality of CBT through supervision at appropriate intervals.

\section{Outcome measures}

We will assess a variety of participant baseline characteristics, including gender, age, education, marital status, employment status, age at pain onset, and duration of pain. Moreover, treatment history will include the names of medications to which the participant has exhibited resistance, current treatment (medication and others) at baseline, other prior treatments, and all changes in conventional treatment throughout the study period.

\section{Primary outcome}

The primary outcome will be the change from baseline to week 16 as indicated by the NRS score. NRS is a self-rated questionnaire and measures pain intensity on a $0-10$ scale $(0=$ nothing to $10=$ severe $)$. Patients keep a pain diary every day. They record (1) maximum pain throughout the day, (2) minimum pain, (3) normal pain as an integer and calculate the weekly average for each pain on the session day (each NRS score = sum total of 1-week NRS score / 7). Numerical values obtained by averaging the values in (1), (2), and (3) are taken as the main evaluation items comprising the composite value of NRS.

\section{Secondary outcomes}

Secondary outcomes will be the change from baseline to week 8 in addition to intensity of each pain as indicated by NRS. Catastrophic perception of pain will be measured with the Pain Catastrophizing Scale 
$(P C S)^{28}$. Degree of daily life impairment will be measured with the Pain Disability Assessment Scale $(\mathrm{PDAS})^{29}$. The Clinical Global Impression of Change scale (CGI-C) is used to evaluate the therapeutic effect caused by the therapist. EQ-5D is the most commonly used scale worldwide for calculating qualityadjusted life years (QALYs), which we will use for the assessment of economic evolution of medical technology ${ }^{30}$. Depressive symptoms will be assessed with the Patient Health Questionnaire-9 (PHQ9) ${ }^{31,32}$ and Beck Depression Inventory $\left.\mathbb{( B D I -} \mathbb{Z}\right)$. Anxiety will be measured with the Generalized Anxiety Disorder scale (GAD-7) ${ }^{33}$. For assessing the satisfaction of the treatment, the Client Satisfaction Questionnaire (CSQ-8) will be used. The assessment items were set based on IMMPACT II "Assessment of Chronic Pain" recommended by the International Pain Society ${ }^{34}$.

\section{Sample size}

With reference to previous studies ${ }^{35,36}$, we assumed that the group difference in NRS variation is 1.67 and the standard deviation is 1.8; the number of subjects was determined by setting the detection power to $80 \%$ and the two-sided significance level to $5 \%$ in the $t$ test. As a result, the number of subjects required per group was estimated to be 20 .

\section{Statistical analysis}

Statistical analysis and reporting of this trial will be conducted in accordance with Consolidated Standards of Reporting Trials (CONSORT) guidelines. Baseline variables will be compared using the Fisher's exact test for categorical outcomes and the unpaired t-test for continuous variables.

For the primary analysis comparing treatment effects, the least-squares means and their $95 \%$ Cls will be estimated by analysis of covariance (ANCOVA) with the change in NRS composite score at week 16 . This ANCOVA model will take into account the variation caused by treatment effects, and the gender and baseline NRS scores ( $\geq 6.3$ and $<6.3$ ) will be entered as covariates. As a sensitivity analysis, we show the transition over time of the NRS scores of each group, confirm the time course measurement data using the linear mixed effect model, and confirm that it is not significantly different from the covariance analysis result.

All comparisons are planned, and all p-values will be two-sided. P-values $<0.05$ will be considered statistically significant. All statistical analyses will be performed by using SAS V.9.4. (SAS Institute, Cary, North Carolina, USA).

\section{Patient and Public Involvement}

Patients or the public are not involved in the design of the study, and will not involve in recruitment and conduction.

Individual results will be collected by a self-writing questionnaire, so that each participant can know changes in their own intervention effects. The degree of satisfaction with the intervention will be assessed by the participant themselves using CSQ-8 (client satisfaction questionnaire), which is 
composed the 1-4 (bad-very good) Likert method. The final research results will be disseminated to study participants on request.

\section{Ethics and Dissemination}

This study will be conducted under the approval of the Institutional Review Board of Chiba University Hospital (approval ID number: G29049). In addition, the Clinical Research Ethics Review Committee will continuously examine whether this test is properly implemented at least once a year. The trial registration number is UMIN000031124.

The patients who wish to participate in this study will be informed of the study objectives and asked again if they are willing to participate. Each patient will be informed that participation is voluntary, and that full anonymity will be provided. Each participant will then be required to provide written informed consent for their participation in this study.

An adverse event could consist of any unfavorable and unintended sign, symptom, or disease temporally associated with this interventional study, whether considered related to the intervention of this study. All adverse events will be reported, and serious adverse events will be immediately reported to the Institutional Review Board of Chiba University Hospital in addition to being registered with the hospital risk management system. Moreover, an independent data monitoring committee will accurately verify detailed records of the clinical study's progress, critical efficacy variables, and safety data, and recommend the sponsor to continue, modify, or terminate the study accordingly.

\section{Discussion}

This study aims to demonstrate the effectiveness of Internet-based CBT with Real-Time Therapist Support Via a Video Conference for patients with chronic pain. The findings of this study will provide a valuable addition to the treatment options for these patients by identifying effective techniques for patients with various types of pain. By using the CBT protocol which we have developed, many therapists will be able to universally provide $\mathrm{CBT}$ of comparable quality in the treatment of chronic pain. There are some limitations to this study. The first is that the quality, cause, and location of pain varies depending on each patient's conditions. Based on this research it is necessary to categorize pain patients and to develop optimal programs for each. The second limitation is that we will be unable to elucidate specific effects of our vCBT program because we will not employ a psychological placebo group to control for non-specific factors. The third limitation is that this study designed a two-armed randomized compared trial by remote Cognitive Behavioral Therapy (VCBT) and usual care alone, so a direct comparison cannot be made with face-to-face CBT. In the future, it will be necessary to conduct a non-inferiority trial of three groups, remote-CBT, face-to-face-CBT, and usual care alone.

\section{Declarations}

Ethics approval and consent to participate 
Ethics approval was granted by the Institutional Review Board of Chiba University Hospital (approval ID number: G29049). All participants will be required to provide written informed consent. Trial registration number: UMIN 000031124; Open public recruiting.

\section{Consent to publication}

Not applicable

\section{Availability of data and materials}

The datasets generated and/or analyzed during the current study are not publicly available due not to locked because of continuing this study but are available from the corresponding author on reasonable request.

\section{Competing interests}

The authors declare that they have no competing interests.

\section{Funding}

This research is supported by the Health and Labor Sciences Research Grant (H29, Refractory, General, 062). The funding body has no role in the design of the study and collection, analysis, and interpretation of data and in writing the manuscript.

\section{Authors' Contributions}

KT and ES contributed to the design of this study and development of the original study protocol. KT was responsible for drafting the initial manuscript. KY established the treatment delivery method, contributing to the outer framework of the study design. NN and KK contributed to creating additional important contents of the manual and critically revised the article.

All authors approved the final version of the manuscript, are accountable for all aspects of the work, and are responsible for resolving any ensuing questions related to the accuracy or integrity of any part of the work.

\section{Acknowledgements}

We thank the Department of Clinical Trials, Chiba University. We would also like to thank Editage (www.editage.com) for English language editing.

\section{Abbreviations}

ANCOVA

Analysis of covariance

ASD 
Autism spectrum disorder

BDI- $\triangle$

Beck Depression Inventory $\mathbb{\Delta}$

CBT

Cognitive Behavioral therapy

CGI-C

Clinical Global Impression of Change scale

CONSORT

Consolidated Standards of Reporting Trials

CSQ-8

Client Satisfaction Questionnaire

DSM-5

Diagnostic and Statistical Manual of Mental Disorders, Fifth Edition

GAD-7

Generalized Anxiety Disorder scale

ICD-11

International Disease Classification version 11

NRS

Numerical Rating Scale

PDAS

Pain Disability Assessment Scale

PCS

Pain Catastrophizing Scale

PHQ-9

Patient Health Questionnaire-9

QALYs

Quality-adjusted life years

QOL

Quality of Life

TAU

Treatment as usual

vCBT

Internet-based Cognitive Behavioral Therapy with Real-Time Therapist Support Via a Video Conference WHO

World Health Organization

\section{References}

1. Nicholas M, Vlaeyen JWS, Rief W, Barke A, Aziz Q, Benoliel R, et al. The IASP classification of chronic pain for ICD-11: chronic primary pain. Pain 2019;160:28-37. doi: 
10.1097/j.pain.0000000000001390.

2. Treede RD, Rief W, Barke A, Aziz Q, Bennett MI, Benoliel R, et al. A classification of chronic pain for ICD-11. Pain 2015;156:1003-7. doi: 10.1097/j.pain.0000000000000160.

3. Dahlhamer J, Lucas J, Zelaya C, Nahin R, Mackey S, DeBar L, et al. Prevalence of chronic pain and high-impact chronic pain among adults - United States, 2016. Centers for Disease Control and Prevention, Morb Mortal Wkly Rep. 2018;67(36):1001-6.

4. de Souza JB, Grossmann E, Perissinotti DMN, de Oliveira Junior JO, da Fonseca PRB, Posso IP. Prevalence of chronic pain, treatments, perception, and interference on life activities: Brazilian population-based survey. Pain Res Manag. doi: 10.1155/2017/4643830 [Published Online First: September 26, 2017].

5. Goldberg DS, McGee SJ. Pain as a global public health priority. BMC Public Health 2011;11:770. doi: 10.1186/1471-2458-11-770.

6. Dale R, Stacey B. Multimodal treatment of chronic pain. Med Clin North Am. 2016;100:55-64. doi: 10.1016/j.mcna.2015.08.012.

7. Morley S, Eccleston C, Williams A. Systematic review and meta-analysis of randomized controlled trials of cognitive behaviour therapy and behaviour therapy for chronic pain in adults, excluding headache. Pain 1999;80:1-13.

8. Knoerl R, Lavoie Smith EM, Weisberg J. Chronic pain and cognitive behavioral therapy: an integrative view. West J Nurs Res. 2016;38:596-628. doi: 10.1177/0193945915615869.

9. Cherkin DC, Sherman KJ, Balderson BH, et al. Effect of mindfulness-based stress reduction vs cognitive behavioral therapy or usual care on back pain and functional limitations in adults with chronic low back pain: a randomized clinical trial. JAMA 2016;315:1240-49.

10. Williams AC, Eccleston C, Morley S. Psychological therapies for the management of chronic pain (excluding headache) in adults. Cochrane Database Syst Rev. 2012;11. doi: 10.1002/14651858.CD007407.pub3 [Published Online First November 14, 2012].

11. Romero-Sanchiz P, Nogueira-Arjona R, Garcia-Ruiz A, Luciano JV, Garcia Campayo J, Gili M, et al. Economic evaluation of a guided and unguided internet-based CBT intervention for major depression: results from a multi-center, three-armed randomized controlled trial. PLoS ONE 2017;12(2):e0172741. doi: 10.1371/journal.pone.0172741 [Published Online First February 27, 2017].

12. Ritterband LM, Thorndike FP, Ingersoll KS, Lord HR, Gonder-Frederick L, Frederick C, et al. Effect of a web-based cognitive behavior therapy for insomnia intervention with 1-year follow-up: a randomized clinical trial. JAMA Psychiatry 2017;74:68-75. doi: 10.1001/jamapsychiatry.2016.3249.

13. Spence SH, Donovan CL, March S, Kenardy JA, Hearn CS. Generic versus disorder specific cognitive behavior therapy for social anxiety disorder in youth: a randomized controlled trial using internet delivery. Behav Res Ther. 2017;90:41-57. doi: 10.1016/j.brat.2016.12.003.

14. de Zwaan M, Herpertz S, Zipfel S, Svaldi J, Friederich HC, Schmidt F, et al. Effect of internet-based guided self-help vs individual face-to-face treatment on full or subsyndromal binge eating disorder in 
overweight or obese patients. JAMA Psychiatry 2017;74:987-995. doi:

10.1001/jamapsychiatry.2017.2150.

15. Friedberg F, Williams DA, Collinge W. Lifestyle-oriented non-pharmacological treatments for fibromyalgia: a clinical overview and applications with home-based technologies. J Pain Res. 2012;5:425-435. doi: 10.2147/JPR.S35199.

16. Palermo TM, Law EF, Fales J, Bromberg MH, Jessen-Fiddick T, Tai G. Internet-delivered cognitivebehavioral treatment for adolescents with chronic pain and their parents: a randomized controlled multicenter trial. Pain 2016;157:174-185. doi: 10.1097/j.pain.0000000000000348.

17. Vigerland S, Lenhard F, Bonnert M, Lalouni M, Hedman E, Ahlen J, et al. Internet-delivered cognitive behavior therapy for children and adolescents: a systematic review and meta-analysis. Clin Psychol Rev. 2016;50:1-10. doi: 10.1016/j.cpr.2016.09.005.

18. Carpenter KM, Stoner SA, Mundt JM, Stoelb B. An online self-help CBT intervention for chronic lower back pain. Clin J Pain 2012;28:14-22. doi: 10.1097/AJP.0b013e31822363db.

19. Ang DC, Jensen MP, Steiner JL, Hilligoss J, Gracely RH, Saha C. Combining cognitive-behavioral therapy and milnacipran for fibromyalgia: a feasibility randomized-controlled trial. Clin J Pain 2013;29:747-54. doi: 10.1097/AJP.0b013e31827a784e.

20. García Carrasco D, Aboitiz Cantalapiedra J. Effectiveness of motor imagery or mental practice in functional recovery after stroke: a systematic review. Neurología 2016;31:43-52. doi: 10.1016/j.nrl.2013.02.003.

21. Cocks M, Moulton CA, Luu S, Cil T. What surgeons can learn from athletes: mental practice in sports and surgery. J Surg Educ. 2014;71:262-9. doi: 10.1016/j.jsurg.2013.07.002.

22. Tsao JW, Finn SB, Miller ME. Reversal of phantom pain and hand-to-face remapping after brachial plexus avulsion. Ann Clin Transl Neurol. 2016;3:463-464. doi: 10.1002/acn3.316.

23. Ramachandran VS, Rogers-Ramachandran D, Cobb S. Touching the phantom limb. Nature 1995;377:489-90. doi: 10.1038/377489a0.

24. Flor H, Nikolajsen L, Staehelin Jensen T. Phantom limb pain: a case of maladaptive CNS plasticity? Nat Rev Neurosci. 2006;7:873-81. doi: 10.1038/nrn1991.

25. Fredrickson BL, Kahneman D. Duration neglect in retrospective evaluations of affective episodes. J Pers Soc Psychol. 1993;65:45-55. doi: 10.1037//0022-3514.65.1.45.

26. Marta IE, Baldan SS, Berton AF, Pavam M, da Silva MJ. The effectiveness of therapeutic touch on pain, depression and sleep in patients with chronic pain: clinical trial. Rev Esc Enferm USP 2010;44:1100-6. doi: 10.1590/s0080-62342010000400035 [in Portuguese].

27. Otis JD. Management chronic pain: A cognitive-behavioral therapy approach, therapist guide. New York: Oxford University Press; 2007.

28. Sullivan MJL. The pain catastrophizing scale: User manual. Montreal: McGill University; 2009.

29. Yamashiro K, Arimura T, Iwaki R, Jensen M, Kubo C, Hosoi M. A multidimensional measure of pain interference: Reliability and validity of the pain disability assessment scale. Clin J Pain 
2011;27(4):338-43. doi: 10.1097/AJP.0b013e318204858a.

30. Tsuchiya A, Ikeda S, Ikegami N, Nishimura S, Sakai I, Fukuda T, et al. Estimating an EQ-5D population value set: The case of Japan. Health Econ. 2002;11(4):341-53. doi: 10.1002/hec.673.

31. Muramatsu K. Patient Health Questionnaire (PHQ-9, PHQ-15) Japanese version and Generalized Anxiety Disorder-7 Japanese version: up to date. Stud Clin Psychol. 2014;7:35-39.

32. Spitzer RL, Kroenke K, Williams JB, The Patient Health Questionnaire Primary Care Study Group. Validation and utility of a self-report version of PRIME-MD: The PHQ primary care study. JAMA 1999;282(18):1737-44. doi: 10.1001/jama.282.18.1737.

33. Spitzer RL, Kroenke K, Williams JB, Löwe B. A brief measure for assessing generalized anxiety disorder: The GAD-7. Arch Intern Med. 2006;166(10):1092-97. doi: 1001/archinte.166.10.1092.

34. Dworkin RH, Turk DC, Wyrwich KW, et. al. Interpreting the clinical importance of treatment outcomes in chronic pain clinical trials: IMMPACT recommendations. J Pain 2008;9:105-21.

35. Carvalho C, Caetano JM, Cunha L, Rebouta P, Kaptchuk TJ, Kirsch I. Open-label placebo treatment in chronic low back pain: a randomized controlled trial. Pain 2016;157:2766-72. doi: 10.1097/j.pain.0000000000000700.

36. Palermo TM, Wilson AC, Peters M, Lewandowski A, Somhegyi H. Randomized controlled trial of an internet-delivered family cognitive-behavioral therapy intervention for children and adolescents with chronic pain. Pain 2009;146:205-13. doi: 10.1016/j.pain.2009.07.034.

\section{Tables}

Due to technical limitations, the tables are only available as a download in the supplemental files section.

\section{Figures}




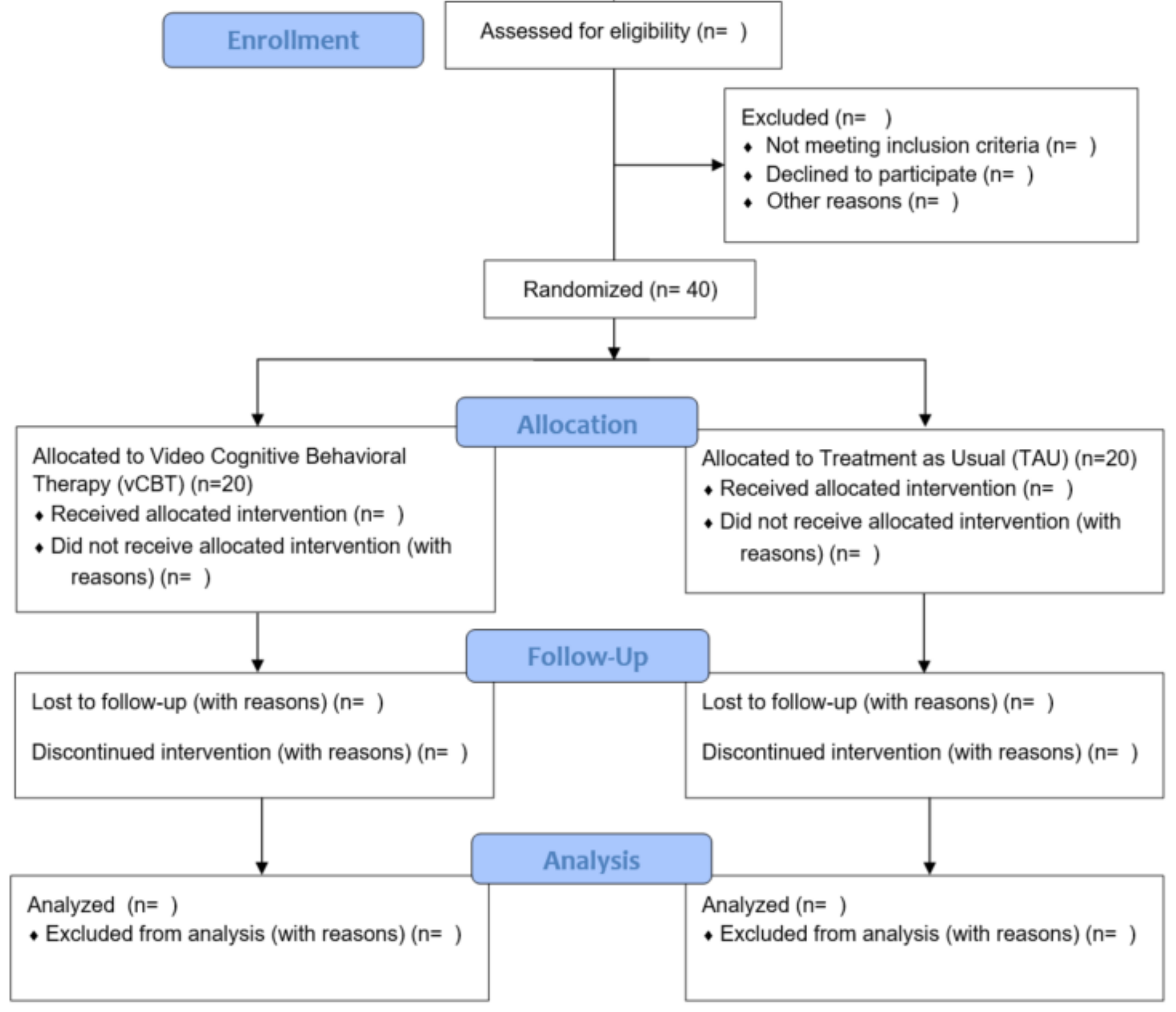

\section{Figure 1}

This study was designed as a prospective randomized open-labelled pilot trial with two parallel 16-week intervention groups: a vCBT group that also received treatment as usual and a continuing TAU group alone

\section{Supplementary Files}


This is a list of supplementary files associated with this preprint. Click to download.

- Table1.xls

- SPIRITChecklistBMCKayokoTaguchi.doc

- Table2.xls 